\title{
Elicitation using multiple price list formats
}

\author{
Steffen Andersen • Glenn W. Harrison • \\ Morten Igel Lau • E. Elisabet Rutström
}

Published online: 13 June 2008

(C) Economic Science Association 2008

\author{
Erratum to: Exp Econ 9, 383-405 (2006) \\ DOI 10.1007/s10683-006-7055-6
}

Table 6 in Andersen, Harrison, Lau and Williams (2006) contains a transcription error for some of the estimates. The columns showing $p$-values and lower and upper $95 \%$ confidence intervals instead show $t$-values, $p$-values and the lower $95 \%$ confidence interval, respectively. The error arose as we substituted estimates from a final run of computer software, to ensure that the software replicated the estimates shown. The exposition in the text is correct, and the correct Table 6 appears below.

The online version of the original article can be found at doi:10.1007/s10683-008-9204-6.

S. Andersen $(\bowtie)$

Centre for Economics and Business Research, Copenhagen Business School, Copenhagen, Denmark e-mail: sa@cebr.dk

G.W. Harrison · E.E. Rutström

Department of Economics, College of Business Administration, University of Central Florida, Orlando, USA

G.W. Harrison

e-mail: gharrison@ research.bus.ucf.edu

E.E. Rutström

e-mail: erutstrom@bus.ucf.edu

M.I. Lau

Department of Economics and Finance, Durham Business School, Durham University, Durham, UK e-mail: m.i.lau@durham.ac.uk 
Table 6 Heteroskedasticity in initial risk aversion responses. Interval regression allowing for multiplicative heteroskedasticity, with the initial CRRA interval chosen by the subject as the dependent variable. Robust standard errors, allowing for clustering on the individual. $N=356$, based on 90 subjects

\begin{tabular}{|c|c|c|c|c|c|c|}
\hline Variable & Description & Estimate & Standard & $\begin{array}{l}p \text {-value } \\
\text { error }\end{array}$ & $\begin{array}{l}\text { Lower } 95 \% \\
\text { confidence } \\
\text { interval }\end{array}$ & $\begin{array}{l}\text { Upper } 95 \% \\
\text { confidence } \\
\text { interval }\end{array}$ \\
\hline Constant & & 0.81 & 0.38 & 0.03 & 0.07 & 1.55 \\
\hline impl & iMPL format & 0.24 & 0.12 & 0.06 & -0.01 & 0.48 \\
\hline smpl & sMPL format & 0.03 & 0.09 & 0.72 & -0.14 & 0.20 \\
\hline skewLO & SkewLO frame & -0.29 & 0.11 & 0.01 & -0.51 & -0.06 \\
\hline skewHI & SkewHI frame & 0.05 & 0.10 & 0.61 & -0.14 & 0.24 \\
\hline Task2 & Second task order & 0.01 & 0.05 & 0.75 & -0.07 & 0.10 \\
\hline Task3 & Third task order & 0.00 & 0.05 & 0.92 & -0.09 & 0.10 \\
\hline Task4 & Fourth task order & 0.09 & 0.04 & 0.03 & 0.01 & 0.18 \\
\hline endowment & Random initial endowment & 0.00 & 0.00 & 0.69 & 0.00 & 0.00 \\
\hline experimenter & Experimenter effect & -0.13 & 0.10 & 0.18 & -0.32 & 0.06 \\
\hline female & Female & 0.10 & 0.08 & 0.21 & -0.06 & 0.26 \\
\hline single & Lives alone & -0.21 & 0.12 & 0.08 & -0.45 & 0.03 \\
\hline nhhd & Number in household & 0.10 & 0.09 & 0.25 & -0.07 & 0.27 \\
\hline owner & Owns home or apartment & -0.05 & 0.15 & 0.74 & -0.36 & 0.25 \\
\hline student & Student & 0.00 & 0.13 & 0.97 & -0.24 & 0.25 \\
\hline skilled & Some post-secondary education & 0.03 & 0.10 & 0.76 & -0.17 & 0.23 \\
\hline longedu & Substantial higher education & 0.02 & 0.12 & 0.84 & -0.21 & 0.25 \\
\hline IncLow & Lower level income & 0.04 & 0.17 & 0.82 & -0.29 & 0.36 \\
\hline IncHigh & Higher level income & -0.14 & 0.20 & 0.49 & -0.53 & 0.25 \\
\hline copen & Lives in Copenhagen area & -0.12 & 0.24 & 0.60 & -0.59 & 0.34 \\
\hline city & $\begin{array}{l}\text { Lives in larger city of } 20,000 \\
\text { or more }\end{array}$ & -0.16 & 0.28 & 0.58 & -0.71 & 0.40 \\
\hline$\sigma_{\text {constant }}$ & Standard deviation of residual & -0.54 & 0.23 & 0.02 & -0.99 & -0.09 \\
\hline$\sigma_{\mathrm{iMPL}}$ & $\begin{array}{l}\text { Multiplicative heteroskedasticity, } \\
\text { iMPL }\end{array}$ & 0.46 & 0.19 & 0.02 & 0.08 & 0.84 \\
\hline$\sigma_{\mathrm{sMPL}}$ & $\begin{array}{l}\text { Multiplicative heteroskedasticity, } \\
\text { sMPL }\end{array}$ & -0.09 & 0.19 & 0.64 & -0.47 & 0.29 \\
\hline$\sigma_{\text {skewLO }}$ & $\begin{array}{l}\text { Multiplicative heteroskedasticity, } \\
\text { skewLO }\end{array}$ & -0.03 & 0.21 & 0.87 & -0.45 & 0.39 \\
\hline$\sigma_{\text {skewHI }}$ & $\begin{array}{l}\text { Multiplicative heteroskedasticity, } \\
\text { skewHI }\end{array}$ & -0.20 & 0.17 & 0.26 & -0.54 & 0.14 \\
\hline$\sigma_{\text {endowment }}$ & $\begin{array}{l}\text { Multiplicative heteroskedasticity, } \\
\text { endowment }\end{array}$ & -0.01 & 0.00 & 0.04 & -0.01 & 0.00 \\
\hline$\sigma_{\text {Task2 }}$ & $\begin{array}{l}\text { Multiplicative heteroskedasticity, } \\
\text { Task2 }\end{array}$ & -0.29 & 0.10 & 0.00 & -0.48 & -0.10 \\
\hline$\sigma_{\text {Task } 3}$ & $\begin{array}{l}\text { Multiplicative heteroskedasticity, } \\
\text { Task3 }\end{array}$ & -0.13 & 0.11 & 0.25 & -0.34 & 0.09 \\
\hline$\sigma_{\text {Task4 }}$ & $\begin{array}{l}\text { Multiplicative heteroskedasticity, } \\
\text { Task4 }\end{array}$ & -0.07 & 0.11 & 0.55 & -0.29 & 0.16 \\
\hline
\end{tabular}

Notes: Log-likelihood value is -443.7 ; Wald test for null hypothesis that all coefficients are zero has a $\chi^{2}$ value of 40.7 with 20 degrees of freedom, implying a $p$-value of 0.004

Legend: Most variables have self-evident definitions, or are defined under Table 3 